\title{
Binding of porcine spermatozoa to uterine epithelial cells modulates the female immune response and might indicate the formation of a pre-oviductal sperm reservoir
}

\author{
U. Taylor ${ }^{1}$, H. Zerbe ${ }^{2}$, H.M. Seyfert ${ }^{3}$, D. Rath ${ }^{1}$ and H.J. Schuberth ${ }^{4}$ \\ 'Institute of Farm Animal Cenetics, Friedrich-Loeffler-Institute, Federal Research Institute for Animal \\ Health, 31535 Neustadt, Cermany; ${ }^{2}$ Clinic for Ruminants, LMU Munich, 85764 Oberschleissheim, \\ Cermany; ${ }^{3}$ Research Institute for the Biology of Farm Animals, 18196 Dummerstorf, Cermany; ${ }^{4}$ institute \\ of Immunology, University of Veterinary Medicine, 30173 Hanover, Cermany
}

Inseminations in pigs are characterized by the tremendous amount of spermatozoa needed for successful fertilisation. If, in contrast, spermatozoa are deposited at the tip of the uterine horn a fraction of the usual porcine insemination dose suffices (Johnson 1991, Vazquez et al. 2005). It thus seems to be the uterine passage where the need for such high sperm numbers arises. In the past the provision of sufficient sperm numbers to reach acceptable fertility rates did not pose a problem due to the abundance of spermatozoa in one single boar ejaculate. However, modern biotechnological procedures, such as sex sorting of spermatozoa, require insemination of sperm portions containing no more then $50 \times 10^{6}$ spermatozoa. To facilitate insemination with such small sperm doses also for conventional Al-techniques, more knowledge has to be gathered about fundamental sperm transport and selection mechanisms within the uterus. Previous studies on the pig uterus (Lovell \& Getty 1968), the utero-tubal junction (Rodriguez-Martinez et al. 1990) and the oviduct (Wagner et al. 2002) suggested that spermatozoa are indeed subject to close interaction and even binding with the epithelial structures of the female genital tract. The present study aimed to further our understanding of such interactions specifically in the porcine uterus.

For this purpose an ex vivo model was developed using uterine segments of $10 \mathrm{~cm}$ derived from 50 freshly slaughtered peri-ovulatory German Landrace gilts. In each segment $100 \times 10^{6}$ spermatozoa were incubated for $60 \mathrm{~min}$ at $38^{\circ} \mathrm{C}$. The sperm cells originated from ejaculates provided by 4 boars of the same breed and of proven fertility. Previous to incubation spermatozoa were either washed and diluted in the semen extender Androhep ${ }^{\text {Tw }}$ or diluted with autologous seminal plasma without further washing. The spermatozoa were subsequently flushed out of the segments, counted and their viability parameters were established flow cytometrically using the stains $\mathrm{Pl}$ and $\mathrm{JC} 1$ for membrane integrity and mitochondrial membrane potential respectively.

The results indicated a retention of viable spermatozoa within the uterine cavity since only $55 \pm 7 \%$ of the intact spermatozoa ( $\mathrm{PI}-/ \mathrm{JCl}+$ ) were rediscovered in the flushing, while the damaged sperm population ( $\mathrm{PI}+/ \mathrm{JC1}-)$ was flushed out almost in its entity $(93 \pm 12 \% ; p<0.03)$. The effect was more emphasised in the sperm population, which had been washed and diluted in Androhep ${ }^{\text {tw }}(p<0.05)$. The location of the uterine segments in relation to the cervix had no effect on the numbers of recovered sperm cells. Neither were differences observed between segments from the right and left uterine horn.

In order to determine the physiological reasons behind the observed uterine sperm retention an in vivo experiment was performed looking for changes in uterine gene expression in response to insemination by measuring endometrial mRNA concentration. Because many processes concerning reproduction such as ovulation and implantation involve the immune system, the

E-mail: ulrike.taylor@fli.bund.de 
mRNAs chosen to be examined encoded for 6 cytokines suggested to have impact on reproductive outcome (GM-CSF, IL-10, CXCL8, TNF- $\alpha$, TGF- $\beta$ \& IL-6) and 2 products of the arachidonic acid metabolism (COX-2, ALOX-5). For the experiment 68 periovulatory gilts received a $90 \mathrm{ml}$ uterine infusion consisting of either Androhep ${ }^{\mathrm{m}}(\mathrm{AH})$ or seminal plasma (SP) with or without spermatozoa. Endometrial tissue samples were taken $3 \mathrm{~h}$ after treatment. The mRNA copy numbers were determined by qRT-PCR and compared with baseline expression obtained from 12 otherwise identically treated non-inseminated sows. Results showed that all chosen genes displayed baseline expression. However, insemination without spermatozoa led to a general up-regulation of all examined genes, which was significant in case of IL-10 (SP: 1.5-fold), CXCL8 (AH: 7.1-fold), TNF- $\alpha$ (AH: 1.9-fold) and COX-2 (AH: 7-fold). Interestingly, despite their majorly different composition Androhep ${ }^{i m}$ and seminal plasma elicited a significantly different response only concerning the arachidonic acid metabolite COX-2. Most surprisingly however was the result that the presence of spermatozoa led to a significant down-regulation back to baseline levels for every mediator tested.

In conclusion, based on the described results we propose the hypothesis that the immense amounts of spermatozoa needed for insemination in pigs is at least partially due to sperm binding sites along the endometrium, which need to be saturated before unbound sperm can proceed to the oviduct. Interestingly, the majority of bound spermatozoa are membrane intact and possess functional mitochondria, i.e. are potential candidates for fertilisation. The exact binding mechanism and the biological consequences remain, however, elusive. The sperm binding sites might serve several purposes. For once, the bound spermatozoa might serve as a secondary reservoir to feed the oviductal reservoir, in case of a delayed ovulation. The fact that bound sperm are viable support this presumption. On the other hand, they could also function as part of a negative selection mechanism, which prevents boars with oligozoospermia to mate successfully, a fact that would have to be overcome for low dose insemination for instance by changing the site of application close to the tip of the uterine horn as described by Vazquez et al. (2005). Furthermore, the observed sperm-associated modulation of uterine gene expression strongly suggests that sperm binding to endometrial cells has a considerable influence on the female immune response. The down-regulation of inflammation-relevant cytokines and arachidonic acid metabolites might serve to tightly control the issuing post-mating inflammatory reaction described in pigs (Matthijs et al. 2003). As a long-term perspective it could also be part of the tolerance induction process against paternal antigens or otherwise set off the preparation of the uterus for the reception of the conceptus.

We gratefully acknowledge the financial support of the Hans Wilhelm Schaumann Foundation and the German Research Foundation.

\section{References}

Johnson, LA 1991 Sex preselection in swine: Altered sex ratios in offspring following surgical insemination of Flow Sorted X-and Y-bearing sperm. Reproduction in Domestic Animals 26 309-314.

Lovell JE \& Getty R 1968 Fate of semen in the uterus of the sow: histological study of endometrium during the 27 hours after natural services. American lournal of Veterinary. Research 29 609-625.

Matthijs A, Engel B \& Woelders H 2003 Neutrophil recruitment and phagocytosis of boar spermatozoa after artificial insemination of sows, and the effects of inseminate volume, sperm dose and specific additives in the extender. Reproduction 125 357-367.
Rodriguez-Martinez H, Nicander L, Viring S, Einarsson S \& Larsson K 1990 Ultrastructure of the uterotubal junction in preovulatory pigs. Anatomy Histology Embryology $1916-36$.

Vazquez IM, Martinez EA, Roca I, Gil MA, Parrilla I, Cuello C, Carvajal G, Lucas X, \& Vazquez IL 2005 Improving the efficiency of sperm technologies in pigs: the value of deep intrauterine insemination. Theriogenology 63 536-547.

Wagner A, Ekhlasi-Hundrieser $M$, Hettel $C$, Petrunkina A, Waberski D, Nimtz M, Toepfer-Petersen E 2002 Carbohydrate-based interactions of oviductal sperm reservoir formation-studies in the pig. Molecular Reproduction Development 61 249-25. 